\section{SAT0468 INFECTIOUS SPONDYLODISCITIS IN A SPANISH REGIONAL HOSPITAL BETWEEN 2000 AND 2018: 66 CASES}

Isla Morante Bolado ${ }^{1}$, Montserrat Santos Gómez ${ }^{1}$, Ignacio Villa-Blanco ${ }^{1}$, Leyre Riancho Zarrabeitia ${ }^{1}$, Elena Aurrecoechea ${ }^{2}$, Teresa Ruiz Jimeno ${ }^{3}$, Jaime Calvo ${ }^{4}$ ${ }^{1}$ Sierrallana Hospital, Torrelavega, Spain; ${ }^{2}$ Sierrallana Hospital, Torrelavega, Spain; ${ }^{3}$ Universitary Hospital of Valdecilla, Santander, Spain; ${ }^{4}$ Universitary Hospital of Araba, Vitoria, Spain

Background: Spondylodiscitis have a large spectrum of clinical manifestations. The early diagnosis and treatment of this condition are essential to give the patient the best chance of a good outcome.

Objectives: To analyze the clinical characteristics, most frequent diagnostic methods and different treatments used in spondylodiscitis (SD) in our sanitary area.

Methods: Descriptive and retrospective study of patients with the diagnose of infectious SD (clinical or microbiological) from 2000 to 2018. In each case we studied the presence of underlying diseases, an episode of infection in the previous 6 months, way of presentation, location, diagnostic methods, treatment and evolution, comparing among different etiologies.

Results: 66 patients were diagnosed of spondylodiscitis. 44 men (24-90 years: mean 71.7). 62 were pyogenic, 3 tuberculous (TBC) SD, and 1 candida. The patients with TBC were younger (mean age: 45.3; $\mathrm{p}<0.05$ ). An underlying disease was observed in 51 patients, specially Diabetes Mellitus (DM) ( $31 \%$ of SD). 4 patients were Rheumatoid Arthritis patients. A previous episode of bacteriemia or a primary source of infection was identify in a $34.8 \%$ of the cases, obtaining a microbiological isolation in 50/66 (75.7\%) SD (46 bacterial, 3 TBC and 1 Candida). The most frequent pathogens were Gram $+(\mathrm{G}+)(51 \%$ of the total $\mathrm{SD})$ being $\mathrm{S}$. aureus and $\mathrm{S}$ epidermidis responsible of $23 / 66$ cases (34.8\%). In the $94 \%$ of $\mathrm{SD}$ caused by $\mathrm{G}_{+}$, hemocultures positive were obtained, in comparison to a $55 \%$ of SD caused by $\mathrm{G}-(\mathrm{p}=0.016)$.

The most frequent presentation symptoms were: lumbar pain $(90 \%)$, fever $(53 \%)$ and neurological deficit (19\%). Leucocytosis was present in only a third of the SD, observing an increase of ESR and CRP in the pyogen etiology ( $p$ no significative for low number of patients in SD group caused by TBC) and lower levels of hemoglobin, cholesterol and albumin. Lumbar area was affected in the $75.7 \%$ of SD $\left(77 \%\right.$ in $\mathrm{G}_{+}$and $50 \%$ in G-). In a $13.6 \%$ of patients, more than one intersomatic space was affected, being visible the presence of an abscess in 47/66 cases $(71.2 \%)$. It was necessary surgical treatment in 10/47 (21.2\%). 5 patients died due to pathology related to SD $(7.5 \%)$, without any correlation with a risk factor and other 5 presented a relapse in the subsequent months. Conclusion: - The bacterial SD are the predominant group, being DM the most frequent risk factor.

- The incidence of SD due to TBC and fungi is scarce in our environment, being absent the Brucella etiology.

- The G+ SD usually have a previous associated bacteriemia.

- The majority of the patients had pain in the presentation, but only half of them had associated fever.

- The most frequent location of SD was lumbar.

- We established a $7.5 \%$ of mortality rate in our sanitary area.

Disclosure of Interests: Isla Morante Bolado: None declared, Montserrat Santos Gómez: None declared, Ignacio Villa-Blanco: None declared, Leyre Riancho Zarrabeitia: None declared, Elena Aurrecoechea: None declared, Teresa Ruiz Jimeno: None declared, Jaime Calvo Consultant for: BristolMyers Squibb, Janssen, Celgene, Sanofi Genzyme, Speakers bureau: Bristol-Myers Squibb

DOI: 10.1136/annrheumdis-2019-eular.3641

\section{SAT0469 \\ THE IMPACT OF CHIKUNGUNYA VIRUS INFECTION ON QUALITY OF LIFE, FUNCTIONAL STATUS AND WORK ABILITY}

Marina Porangaba ${ }^{1}$, André M. Siqueira ${ }^{2}$, Geraldo Castelar' ${ }^{1}{ }^{1}$ Universidade do Estado do Rio de Janeiro, Rio de Janeiro, Brazil; ${ }^{2}$ Fundação Oswaldo Cruz Fiocruz, Rio de Janeiro, Brazil

Background: Chikungunya virus (CHIKV) is a reemergent arbovirus from the Alphavirus genus transmitted by Aedes species mosquitoes. The main feature of CHIKV disease is severe polyarthralgia, which is reported in more than $90 \%$ of cases. Musculoskeletal symptoms may persist in the subacute ( $>3$ weeks) and chronic ( $>12$ weeks) phases, causing critical physical impairment and significantly impacting the quality of life of patients.
Objectives: To assess the impact of CHIKV on pain, functional status, work ability and health-related quality of life (HRQoL) in the subacute and chronic phases of the disease.

Methods: Patients with a diagnosis of CHIKV disease (confirmed by PCR or serology) with persistent musculoskeletal symptoms after 4 weeks were referred to the Rheumatology outpatient clinic and followed up from Apri 2018 to January 2019. Evaluation questionnaires of pain (visual analogue scale - VAS), disability (Health Assessment Questionanaire - HAQ), HRQOL (Short-form 12 - SF-12) and work ability (Work Productivy and Activity Impairment-WPAI) were applied. The assessments were divided into 3 stages according to the time of disease: subacute from 4 to 12 weeks, chronic from 12 to 24 weeks and chronic with more than 24 weeks.

Results: Of the 69 patients analyzed, $76.81 \%$ were women, mean age $49.78 \pm 14.24$ years, $49.27 \%$ had some comorbidity (such as hypertension and diabetes), 26 were obese and $37.68 \%$ presented a previous musculoskeletal condition. Fifty-eight patients initiated follow-up in the subacute phase, in which the average pain was $6.84 \pm 1.9$, mean $\mathrm{HAQ}$ of $1.59 \pm 0.57$, mean Physical Health Composite Scale Score (PCS) of 26.81 \pm 14.3 and Mental Health Composite Scale Score (MCS) of $36.77 \pm 15.9$. Of the 58 patients, 35 were employed and of these, $54.25 \%$ were absent from work during the previous week. Women presented lower scores in the mental component of SF-12 $(p=0.0215)$ and the presence of comorbidity was related to higher values reported in the pain VAS $(p=0.026)$ In the chronic phase of 12-24 weeks, 50 patients were evaluated, with mean pain $5.27 \pm 2.22$, HAQ $1.16 \pm 0.61$, PCS of $38.82 \pm 17.26$ and MCF 43.72 \pm 17.13$)$. Thirty-two patients were employed, of which $25 \%$ were absent the antecedent week. During this stage, women presented lower values of MCS $(\mathrm{p}=0.0245)$ and the presence of obesity was related to higher values in HAQ $(p=0.0157)$. Finally, in the evaluation of the chronic phase after 24 weeks of evolution, we included 25 patients with the mean of pain $5.64 \pm 2.3$, HAQ $1.11 \pm 0.49$, PCS $36.72 \pm 19$ and MCS $41.56 \pm 17.74$. Fifteen of the 25 patients were employed and $33 \%$ were absent from work the previous week. There was no significant difference between the groups evaluated after 6 months of evolution.

Conclusion: In this study, we demonstrated that the impact of chikungunya persists after 1 month of evolution in a large number of patients The incapacity for work caused by the disease, represented by persistent rates of absenteeism at work in an economically active age group, further aggravates the magnitude of the problem.

\section{REFERENCES}

[1] Couturier, E., Guillemin, F., Mura, et.al. Impaired quality of life after chikungunya virus infection: a 2-year follow-up study. Rheumatology. $2012 \mathrm{Jul}$; 51(7), 1315-22.

[2] Soumahoro, M.-K., Gérardin, P., Boëlle, P.-Y. Et al. Impact of Chikungunya Virus Infection on Health Status and Quality of Life: A Retrospective Cohort Study. PLoS ONE. 2009 Nov; 4(11), e7800.

Disclosure of Interests: None declared

DOI: 10.1136/annrheumdis-2019-eular.6234

\section{SAT0470 RISK OF HOSPITAL ADMISSION DUE TO INFECTION IN PATIENTS UNDERGOING TREATMENT WITH BIOLOGICAL THERAPY: CASE-CONTROL STUDY}

Jose Rosas ${ }^{1,1}$, Ana Pons ${ }^{1}$, José Alberto García-Gómez ${ }^{2}$, José Miguel SenabreGallego $^{1}$, Gregorio Santos Soler ${ }^{1}$, Esteban Salas-Heredia ${ }^{1}$, José Antonio BernalVidal ${ }^{1}$, Catalina Cano ${ }^{1}$, Estíbaliz Ivars ${ }^{1}$, Xavier Barber $^{3}$, AIRE-MB. ${ }^{1}$ Hospital Marina Baixa, Rheumatology, Villajoyosa (Alicante), Spain; ${ }^{2}$ University General Hospital, Infectious Diseases, Elche (Alicante), Spain; ${ }^{3}$ Miguel Hernández University, Statistics, Elche (Alicante), Spain

Objectives: To know risk of admission due to infection in patients treated with biological drugs (DMARDb).

Methods: Case-control study, in patients admitted for infection, treated with DMARDb, from 2000-2018. As a control group, patients included, in treatment with conventional synthetic DMARDs (DMARDcs).

General data (age, gender), disease (diagnosis, time evolution, DMARDb, time in DMARDb, DMARDcs); and infection (time in DMARDb to infection, location of infection, more than one admission).

Results: Of 364 patients, 48 (13\%) admitted for infection: $32(9 \%)$ treated with DMARDb, $16(4 \%)$ with DMARDcs alone. When comparing patients admitted vs who do not, RA predominates and no differences in the treatment with DMARDb and/or DMARDcs, corticosteroids, type of DMARD or the infection. 